\section{Radiologische Spitzenforschung gegen Krebs}

Im Rahmen der Nationalen Dekade gegen Krebs fördert das Bundesministerium für Bildung und Forschung (BMBF) insgesamt 13 Vergleichs- und Optimierungsstudien mit insgesamt bis zu 62 Millionen Euro. Die Radiologie ist mit 2 Forschungsprojekten beteiligt.

Das BMBF hat im Jahr 2019 die „Nationale Dekade gegen Krebs" initiiert mit dem Ziel, Krebserkrankungen möglichst zu verhindern, Heilungschancen durch neue Therapien zu verbessern sowie die Lebenszeit und -qualität von Betroffenen zu erhöhen. Zu den ersten konkreten Maßnahmen der Initiative gehört die erstmalige gezielte Förderung von klinischen Vergleichs- und Optimierungsstudien zur Prävention, Diagnose und Therapie von Krebserkrankungen. Hierfür hat das BMBF bis zu 62 Millionen Euro bereitgestellt. Die Förderung umfasst nicht nur die Durchführung der Studien, sondern auch deren Vorbereitung. Durch ein internationales Gremium unter Einbeziehung von Patientenvertreterinnen und -vertretern sind insgesamt 13 Projekte für die Vorphase ausgewählt worden. Diese haben bis zu 7 Monate Zeit, um ihre Studien zu konzipieren und zu planen, bevor in einer zweiten Begutachtungsrunde über eine weitere Förderung entschieden wird.

Die ausgewählten Projekte decken nicht nur die Bereiche Prävention, Diagnostik, Therapie und Nachsorge, sondern auch verschiedene Behandlungsmethoden ab. Dazu gehören Interventionen in den klassischen Bereichen Operation, Radio- und Chemotherapie, aber auch Psychoonkologie und Sport. Neben Projekten, die die sehr häufig auftretenden Krebserkrankungen der Brust, der Prostata oder des Darms adressieren, wurden ebenfalls Projekte ausgewählt, die sich mit Tumoren der Haut, der Niere oder der Speiseröhre beschäftigen.

\section{Radiologie hervorragend repräsentiert}

Mit ABREMAS und PRIMA zählen gleich 2 aus der Radiologie heraus initiierte bzw. mitinitiierte Studien zu den Forschungsprojekten, die vom BMBF gefördert werden.

\section{ABREMAS - optimierte Früherkennung von Brustkrebs}

Unter Federführung von Univ.-Prof. Dr. Christiane Kuhl, Direktorin der Klinik für Radiologie an der RWTH Aachen, hat sich ein hochkarätig besetztes interdisziplinäres Konsortium unter Einbindung von Vertreterinnen verschiedener Patienteninitiativen zusammengefunden, um im Rahmen der groß angelegten randomisierten klinischen Studie ABREMAS (ABbreviated Breast MRI for Risk-Adjusted Screening: A Prospective Randomized Controlled Clinical Trial) erstmals eine modifizierte Magnetresonanzuntersuchung der Brust, die sogenannte „fokussierte MRT“, mit dem diagnostischen Standard, also der digitalen Mammografie, zu vergleichen und die Praktikabilität und Leistungsfähigkeit dieser verkürzten BrustMRT als alternative Früherkennungsstrategie zu bestimmen. Die Studie adressiert Frauen im Alter zwischen 52 und 69 Jahren, die am Mammografie-Screening teilnehmen und die ausweislich der jeweils letzten Mammografie ein besonders dichtes Brustdrüsengewebe haben.

Weiterführende Informationen zur ABREMAS-Studie finden Sie bei der Nationalen Dekade gegen Krebs.

\section{PRIMA - Diagnoseverfahren}

\section{bei Prostatakrebs}

Die sichere Diagnostik eines Prostatakarzinoms, bei der gleichzeitig unnötige Biopsien, Nebenwirkungen sowie Über- oder Unterbehandlungen vermieden werden können, ist Gegenstand der großen Multicenterstudie PRIMA, die am Universitätsklinikum Düsseldorf von Dr. Rouvier Al-Monajjed, Klinik für Urologie, und PD Dr. Lars Schimmöller, Institut für Diagnostische und Interventionelle Radiologie, entwickelt wurde. Die Studie vergleicht multizentrisch an 6 Universitätskliniken in Nordrhein-Westfalen in Zusammenarbeit mit dem Deutschen Krebsforschungszentrum (DKFZ) das bisherige Standardverfahren in der Prostatakarzinomfrüherkennung bei Männern im Alter zwischen 50 und 75 Jahren mit einer reduzierten, jedoch gezielteren Diagnostik mittels multiparametrischer Magnetresonanztomografie (mpMRT). Gezeigt werden soll, ob mit dieser Methode ebenso viele klinisch relevante (potenziell lebenslimitierende) Prostatakarzinome erkannt bzw. bei negativem Befund mit so hoher Sicherheit ausgeschlossen werden können, dass Männer, bei denen aktuell zur Absicherung der Diagnose noch Gewebeproben aus der Prostata entnommen werden, ggf. gar nicht mehr biopsiert werden müssen. An der Projektentwicklung waren neben den Universitätskliniken auch niedergelassene Urologen, Hausärzte im Rheinland und der Bundesverband Prostatakrebs-Selbsthilfe (BPS) beteiligt.

Weiterführende Informationen zur PRIMAStudie finden Sie bei der Nationalen Dekade gegen Krebs. 